\title{
Web report Nutrition for critically ill patients
}

\author{
Neill Adhikari
}

Lecturer, Department of Critical Care Medicine, Sunnybrook and Women's College Health Sciences Centre, Toronto, Ontario, Canada

Corresponding author: Neill Adhikari, neill.adhikari@sw.ca

Reported: 27 July 2004

Published online: 10 August 2004

This article is online at http://ccforum.com/content/9/3/E10

(c) 2004 BioMed Central Ltd

Website Critical Care Nutrition website

URL http://www.criticalcarenutrition.com

Cost Free

Keywords critical care, nutrition

Nutrition is an integral component of general care for critically ill patients, and numerous randomized trials have addressed research questions related to the optimal feeding route, formulation, dose, and timing of initiation. The mission of the free website www.criticalcarenutrition.com is to improve the practice of nutrition support in the critical care setting through knowledge translation', with specific objectives for knowledge generation, synthesis and translation. Most of the website's resources are devoted to the dissemination of a recently published multidisciplinary evidence-based clinical practice guideline (CPG) on nutritional support for mechanically ventilated critically ill adults [1]. The guideline was peer funded and developed with the participation of multiple academic, professional society, and industry stakeholders. The related website is operated by Critical Care Nutrition, a group of Canadian intensivists and dietitians with diverse backgrounds (intensive care, surgery, nutrition, clinical epidemiology).

For practicing clinicians, including intensive care unit (ICU) directors and dietitians, the most useful website features are the Tools \& Training Kit (designed to facilitate local CPG implementation) and the Nutrition Survey. Implementation tools include a training manual (text and flow diagrams that highlight important CPG recommendations), sample order sets for glycemic control and enteral feeding, printable summary pocket cards, and tips on organizing an implementation workshop. Nine slide presentations on various nutrition topics (e.g. 'Optimizing the Benefits and Minimizing the Risk of Enteral Nutrition' and 'Nutritional Support in Severe Acute Pancreatitis') are available as zipped PowerPoint files and can be modified for local use. Overall, the materials are comprehensive, practical, and novel. They
Critical Care 2005, 9:E10 (DOI 10.1186/cc2929)

are especially appealing given the traditional ineffective approach of relying on journal publication as the primary method of knowledge translation. However, an enthusiastic but administratively inexperienced intensivist or dietitian may find it extremely challenging to build a nutritional quality improvement program using the website tools. Additional useful information would include the following: a specific 'how-to' manual for implementing the CPG (which might include a downloadable lecture, a sample project timeline, and estimated personnel, time and monetary requirements); links to general change management resources; and evidence of usability testing of the implementation tools to match the rigor of the CPG development process.

The Nutrition Survey is a quality improvement tool that an ICU can use initially to compare its nutrition practice to that in other centres and subsequently to assess the impact of a quality improvement initiative on adherence to best practices. Patient data are entered on the website either directly or after initial data entry on downloadable printable forms. Local experience with the survey has been positive, with straightforward, rapid, and reliable web data entry. The ICU must enter data on at least 10 patients in order to receive a report. Prospective participants can examine the manual data collection forms and help information, but there is no information on the length of time required for data entry, sample reports, or the number and experience of participating hospitals to date.

Other useful features of the site are a list of planned and ongoing nutritional support studies initiated by the Critical Care Nutrition group and a list of upcoming conferences

$\mathrm{CPG}=$ clinical practice guideline; $\mathrm{ICU}=$ intensive care unit. 
(although at the time of this review the three conferences listed had already taken place). Of note, one recently completed randomized trial evaluated the effectiveness of the website's implementation tools as compared with passive CPG dissemination.

The website is functional, with rapid page loading and downloads, easy navigation, working links to nutrition and critical care professional organizations, and no advertisements. I saved some files to my hard drive because they would not open directly. The website does not disclose funding sources but it does not endorse or advertise specific products. There are plans to update the CPG as new evidence becomes available. Other objectives, such as developing a warehouse of randomized trials of nutritional interventions, remain to be implemented. Overall, this site provides intensivists, dietitians, and others interested in nutritional support of the critically ill with a rich set of tools to help translate evidence-based recommendations into practice.

\section{Best feature}

The best feature is the provision of a downloadable comprehensive scientifically rigorous evidence-based CPG for nutrition support, along with associated implementation tools.

\section{Worst feature}

Despite the tools, there may not be enough practical information to enable clinicians to initiate and sustain a nutrition quality improvement initiative.

\section{Wish list}

Of great use would be a step-by-step 'how-to' manual to assist guideline implementation; presentation of the guideline in HTML format with links to primary studies and graphics; and audio to accompany the presentations.

\section{Other links}

Institute for Healthcare Improvement - http://www.ihi.org/ihi This website provides information and resources on quality improvement in health care, including critical care. The website is free, but some education modules require purchase.

\section{Competing interests}

None declared.

\section{Acknowledgement}

I thank Andrea Chan, RD CNSD, for providing feedback on data entry for the Nutrition Survey.

\section{Reference}

1. Heyland DK, Dhaliwal R, Drover JW, Gramlich L, Dodek P, Canadian Critical Care Clinical Practice Guidelines Committee: Canadian clinical practice guidelines for nutrition support in mechanically ventilated, critically ill adult patients. JPEN J Parenter Enteral Nutr 2003, 27:355-373. 